\title{
Fractura de elastómeros EPDM cargados con microesferas de vidrio mediante el trabajo esencial de fractura
}

\author{
J. I. VELASCO ${ }^{1}$, D. ARENCÓN ${ }^{2} M^{\mathrm{a}}$. L. MASPOCH ${ }^{1}$ C. MORHAIN ${ }^{2}$ \\ ${ }^{1}$ Departament de Ciències dels Materials i Enginyeria Metal.lúrgica. Universitat Politècnica de Catalunya (UPC). ETSEIB. 08028 Barcelona \\ ${ }^{2}$ Centre Català del Plàstic (CCP). C/ Colom 114, 08222 Terrassa (Barcelona).
}

\begin{abstract}
En el presente trabajo se ha aplicado el concepto de trabajo esencial de fractura (EWF) a una serie de compuestos de EPDM y microesferas de vidrio, sin tratamiento superficial y tratadas con silanos. Se ha comprobado que esta teoría no es aplicable al EPDM puro debido a su elevado carácter elástico, pudiéndose en cambio aplicar a sus compuestos cargados con microesferas de vidrio, pues la presencia de estas partículas induce cierta plasticidad en el EPDM. Los resultados indican que una mayor adhesión entre partícula y matriz se traduce en un mayor valor de trabajo plástico de fractura, así como en una mayor inestabilidad final en la propagación de la grieta. Asimismo se ha estudiado la influencia de la temperatura de procesado sobre las características mecánicas de estos materiales, observándose una caída acusada de la rigidez y de la resistencia a la tracción en todos los compuestos a partir de una temperatura de procesado de $200{ }^{\circ} \mathrm{C}$. La mayor adhesión que se consigue entre la matriz de EPDM y las microesferas de vidrio mediante el tratamiento superficial con silanos se ha confirmado a través de la microscopía electrónica de barrido.
\end{abstract}

Palabras clave: EPDM, microesferas de vidrio, agentes de acoplamiento tipo silano, fractura, trabajo esencial de fractura

Fracture of EPDM filled with glass beads applying the essential work of fracture

In this work, the essential work of fracture concept (EWF) has been applied to untreated and silane- treated glass bead-filled EPDM. It has been proved that this theory is not applicable to pure EPDM due to its high elastic character, but being possible in glass bead-filled EPDM, thus the presence of these particles induces certain plasticity on the EPDM. The results show that the higher adhesion between matrix and particles, the higher value of plastic work of fracture, and the higher final instability of crack propagation. Moreover the influence of processing temperature on the mechanical characteristics of these materials has been studied, noticing a marked drop of stiffness and strength from a temperature processing of $200{ }^{\circ} \mathrm{C}$. The higher adhesion obtained between EPDM matrix and glass beads through surface treatment has been confirmed by scanning electron microscopy.

Keywords: EPDM, glass beads, silane coupling agents, fracture, essential work of fracture

\section{INTRODUCCIÓN}

La teoría del trabajo esencial de fractura (EWF), propuesta por Broberg (1), postula que la energía total de fractura $\left(\mathrm{W}_{\mathrm{f}}\right)$ de una probeta entallada sometida a un esfuerzo de tracción puede dividirse en dos partes: el trabajo esencial de fractura $\left(\mathrm{W}_{\mathrm{e}}\right)$ y el trabajo no esencial de fractura $\left(\mathrm{W}_{\mathrm{p}}\right)$. Al primero se le asocia con la inestabilidad en la punta de la grieta (donde tiene lugar el proceso real de fractura) siendo proporcional a la superficie de ligamento. El segundo se refiere al trabajo de deformación plástica, y es proporcional al volumen de zona exterior de proceso (Fig. 1):

$$
W_{f}=W_{e}+W_{p}=w_{e} l t+w_{p} \beta 1^{2} t
$$

En esta ecuación $\mathrm{w}_{\mathrm{e}} \mathrm{y} \mathrm{w}_{\mathrm{p}}$ representan el trabajo específico esencial y no esencial respectivamente, 1 la longitud de ligamento, $t$ el espesor de la probeta y $\beta$ un factor de forma relacionado con la geometría de la zona plástica. Si dividimos la anterior expresión por el área de ligamento se obtiene la siguiente expresión del trabajo específico de fractura:

$$
\mathrm{w}_{\mathrm{f}}=\mathrm{w}_{\mathrm{e}}+\beta \mathrm{w}_{\mathrm{p}} 1
$$

Por tanto, existe una relación lineal entre el trabajo específico de fractura y la longitud de ligamento que permite obtener un valor de $\mathrm{w}_{\mathrm{e}}$ cuando extrapolamos los resultados a longitud de ligamento cero.

Estas expresiones están condicionadas a un estado tensional puro de tensión plana. Para comprobarlo, se suele utilizar el criterio de Hill (2), que establece que la máxima tensión $\left(\sigma_{\text {net }}\right)$ que se alcanza en el ensayo de fractura (con probetas DDENT) es 1.15 veces la tensión de cedencia $\left(\sigma_{\mathrm{y}}\right)$ del material medida a la misma velocidad de deformación. Assimismo, establece que se alcanza un estado de deformación plana cuando la tensión máxima que se alcanza en el ensayo es superior a 2.97 veces la tensión de cedencia.

Este método de caracterización de la fractura se viene aplicando con éxito a materiales poliméricos en forma de película o plancha delgada (3-6), aunque inicialmente se desarrolló para materiales metálicos (7-8). Si bien la teoría no es aplicable a materiales puramente elásticos (sino a materiales elastoplásticos) algunos autores la han aplicado recientemente con éxito a polipropilenos elastoméricos (9) y a polipropileno modificado con elastómeros termoplásticos y cargas rígidas (10). Nuestro principal objetivo en este trabajo ha sido verificar la 
aplicabilidad de la teoría del trabajo esencial de fractura a compuestos de EPDM y microesferas de vidrio, así como observar los efectos debidos al tratamiento superficial de las microesferas con silanos organofuncionales y de la temperatura de procesado o composición de los materiales.

\section{PROCEDIMIENTO EXPERIMENTAL}

\subsection{Materiales, composición y probetas}

Se eligió un grado comercial de EPDM (Dutral TER4038) suministrado por Enichem que contiene un $27 \%, 68.5 \%$ y $4.5 \%$ de polipropileno, etileno y etilidén-norborneno respectivamente. Las microesferas de vidrio, de tamaño medio de partícula $10 \mu \mathrm{m}$, nos fueron suministradas por Sovitec Ibérica, S.A. Los agentes de acoplamiento utilizados para el tratamiento superficial de las microesferas de vidrio fueron, por un lado, el silano Z-6032 (N-(2-(vinilbencilamino)-etil)-3-aminopropiltrimetoxisilano, Dow Corning) y por otro lado una mezcla de silanos basados en sistemas vinílicos Ucarsil PC-1A y PC-1B (Union Carbide) en proporción 3 a 1.

El tratamiento superficial de las microesferas se realizó de la siguiente manera: se preparó una solución que contenía $30 \mathrm{ml}$ de silano Z-6032, $250 \mathrm{ml}$ de metanol, $60 \mathrm{ml}$ de agua destilada y $5 \mathrm{ml}$ de ácido acético por cada $1.5 \mathrm{~kg}$ de microesferas; dicha solución se agitó durante 20 minutos para asegurar la hidrólisis de los grupos alkoxi del silano, antes de humedecer las microesferas con esta solución. Una vez tratadas se secaron en una estufa a $60^{\circ} \mathrm{C}$ durante 24 horas. Se siguió un procedimiento similar con la mezcla Ucarsil.

La composición de los materiales se llevó a cabo utilizando una extrusora corrotatoria de doble husillo (Collin). El diámetro del husillo fue de $24 \mathrm{~mm}$ y su relación L/D 25. El proceso se llevó a cabo con diferentes perfiles de temperaturas. Como resumen, las temperaturas de boquilla fueron: 115, 150, 180, $200,220,235^{\circ} \mathrm{C}$. La velocidad de giro del husillo se fijó en 100 rpm, consiguiéndose una óptima dispersión de las microesferas en la matriz de EPDM gracias a los elementos de mezcla y a la configuración de doble husillo de la extrusora. Se adaptó una boquilla de sección circular de $3 \mathrm{~mm}$ de diámetro, y el extruído así obtenido se enfrió en un baño de agua y fue granceado. De esta manera, se prepararon 3 compuestos con una concentración nominal de microesferas del $50 \%$ en peso. La nomenclatura de los compuestos preparados es la siguiente: B50 para el compuesto con las microesferas sin tratamiento superficial, BUC50 para las tratadas con la mezcla de silanos Ucarsil y BZ50 para las tratadas con el silano Z-6032. El EPDM no cargado fue sometido también al mismo proceso de extrusión, para someterle a la misma historia térmica y mecánica que los compuestos.

A continuación se moldearon placas de dimensiones $150 \mathrm{~mm}$ x $150 \mathrm{~mm}$ x $1.3 \mathrm{~mm}$ y $150 \mathrm{~mm}$ x $150 \mathrm{~mm}$ x $2.5 \mathrm{~mm}$, por moldeo por compresión, con una prensa de platos calientes a una temperatura de $115^{\circ} \mathrm{C}$ y una presión máxima de 100 bar. Se prepararon dos tipos de probetas a partir de las placas moldeadas. Por un lado, se troquelaron probetas halterio (tipo IV, según ASTM D-412) de las placas de $2.5 \mathrm{~mm}$ de espesor para los ensayos de tracción, y por otro lado se prepararon probetas DDENT de dimensiones $100 \mathrm{~mm}$ x $60 \mathrm{~mm}$ x $1.3 \mathrm{~mm}$, para la aplicación del EWF, de acuerdo con las recomendaciones de la ESIS (11). Las entallas se realizaron con una cuchilla de afeitar, midiéndose la longitud de ligamento con un microscopio óptico.

\subsection{Ensayos}

La caracterización mecánica se realizó mediante ensayos de tracción a una velocidad de desplazamiento de mordazas de $100 \mathrm{~mm} / \mathrm{min}$, a temperatura ambiente, en una máquina universal de ensayos (Adamel DY-30), dotada de una célula de carga de $100 \mathrm{~N}$ y equipada con un extensómetro láser (Hounsfield 500-L). Se ensayaron un mínimo de 5 probetas por cada compuesto y temperatura de procesado. Con las curvas tensión-deformación de cada probeta se pudo determinar tanto el módulo de Young (E), como la resistencia del material (a una deformación del 200\%).

La teoría del trabajo esencial de fractura se aplicó con los resultados obtenidos a partir de ensayos de tracción realizados sobre las probetas DDENT (Fig. 1) a una velocidad de despla-

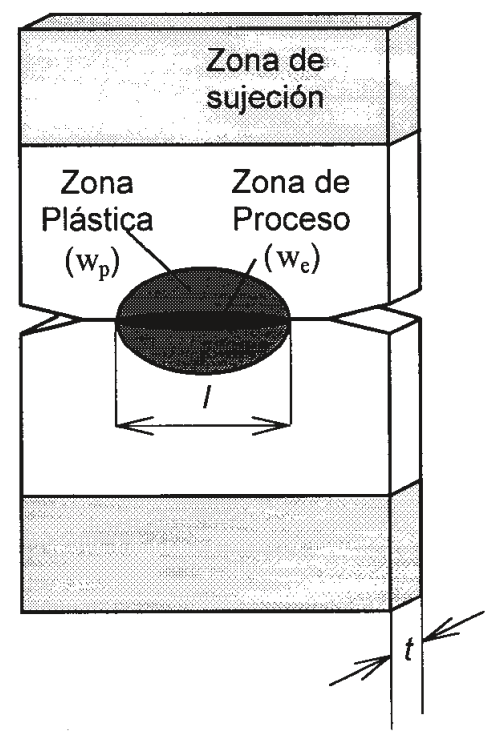

Figura 1. Geometría DDENT empleada en los ensayos EWF.

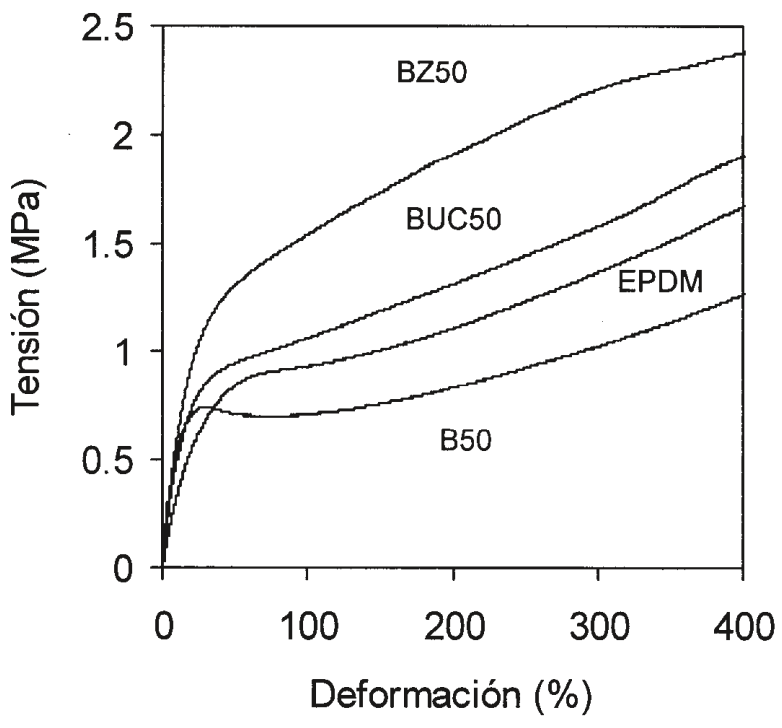

Figura 2. Curvas tensión-deformación comparativas, obtenidas por tracción para los materiales procesados a $150^{\circ} \mathrm{C}$. 
TABla I. VALORES DEL MÓDULO DE YOUNG Y DE LA RESISTENCIA A LA TRACCIÓN DETERMINADOS A 100 MM/MIN PARA TODOS LOS MATERIALES Y TEMPERATURAS DE PROCESADO

\begin{tabular}{|c|c|c|c|}
\hline Material & $\begin{array}{c}\text { Temperatura } \\
\text { de procesado }\left({ }^{\circ} \mathrm{C}\right)\end{array}$ & $\begin{array}{c}\text { Módulo de Young } \\
\text { (MPa) }\end{array}$ & $\begin{array}{r}\text { Resistencia al } \\
200 \% \text { (MPa) }\end{array}$ \\
\hline EPDM & $\begin{array}{l}115 \\
150 \\
180 \\
200 \\
220 \\
235\end{array}$ & $\begin{array}{l}3.79 \pm 0.66 \\
4.10 \pm 0.58 \\
3.66 \pm 0.81 \\
3.75 \pm 0.66 \\
3.26 \pm 0.03 \\
1.87 \pm 0.12\end{array}$ & $\begin{array}{l}1.07 \pm 0.02 \\
0.99 \pm 0.03 \\
1.02 \pm 0.05 \\
0.93 \pm 0.01 \\
1.12 \pm 0.08 \\
0.88 \pm 0.07\end{array}$ \\
\hline B50 & $\begin{array}{l}115 \\
150 \\
180 \\
200 \\
220 \\
235\end{array}$ & $\begin{array}{l}7.67 \pm 0.72 \\
6.26 \pm 0.43 \\
7.74 \pm 0.44 \\
7.11 \pm 0.40 \\
4.83 \pm 0.12 \\
3.48 \pm 0.02\end{array}$ & $\begin{array}{l}1.24 \pm 0.04 \\
0.76 \pm 0.03 \\
0.84 \pm 0.03 \\
0.78 \pm 0.02 \\
0.85 \pm 0.05 \\
0.61 \pm 0.03\end{array}$ \\
\hline BUC50 & $\begin{array}{l}115 \\
150 \\
180 \\
200 \\
220 \\
235\end{array}$ & $\begin{array}{l}7.61 \pm 0.43 \\
6.13 \pm 1.20 \\
6.36 \pm 0.64 \\
7.22 \pm 0.79 \\
5.64 \pm 0.47 \\
4.83 \pm 0.37\end{array}$ & $\begin{array}{l}1.28 \pm 0.16 \\
1.24 \pm 0.05 \\
1.14 \pm 0.01 \\
1.05 \pm 0.08 \\
0.99 \pm 0.04 \\
0.72 \pm 0.07\end{array}$ \\
\hline BZ50 & $\begin{array}{l}115 \\
150 \\
180 \\
200 \\
220 \\
235\end{array}$ & $\begin{array}{l}8.18 \pm 0.44 \\
7.31 \pm 0.35 \\
8.18 \pm 0.44 \\
7.05 \pm 0.74 \\
5.36 \pm 0.66 \\
4.68 \pm 0.28\end{array}$ & $\begin{array}{l}2.00 \pm 0.01 \\
1.89 \pm 0.04 \\
1.61 \pm 0.08 \\
1.75 \pm 0.06 \\
1.48 \pm 0.02 \\
1.08 \pm 0.04\end{array}$ \\
\hline
\end{tabular}

zamiento de mordazas de $10 \mathrm{~mm} / \mathrm{min}$ y temperatura ambiente. Se registraron las curvas fuerza-desplazamiento y la energía total absorbida por la muestra durante el ensayo, calculada como el área bajo dicha curva.

Para investigar los aspectos morfológicos asociados con el proceso de fractura, las superficies de fractura generadas en los ensayos descritos anteriormente se examinaron por microscopía electrónica de barrido (SEM), utilizando un microscopio electrónico Jeol JSM-820, después de cubrir las muestras con una fina capa de oro para conseguir una óptima conductividad.

\section{RESULTADOS Y DISCUSIÓN}

\subsection{Comportamiento a tracción}

Un ejemplo de las curvas tensión-deformación obtenidas se muestra en la Fig. 2 y los valores del módulo de Young (E) y de la resistencia a la tracción $\left(\sigma_{200 \%}\right)$ obtenidos aparecen recogidos en la Tabla I.

Puede apreciarse como la incorporación de una fase rígida, como son las microesferas de vidrio, provoca en el polímero un esperado aumento de rigidez, observándose una tendencia más o menos estable del valor del módulo de Young para todos los compuestos hasta una temperatura de procesado de $20{ }^{\circ} \mathrm{C}$, a partir de la cual se aprecia una notable caída en dicho valor. Por su parte no parecen observarse diferencias significativas entre los valores de $\mathrm{E}$ con las microesferas tratadas con
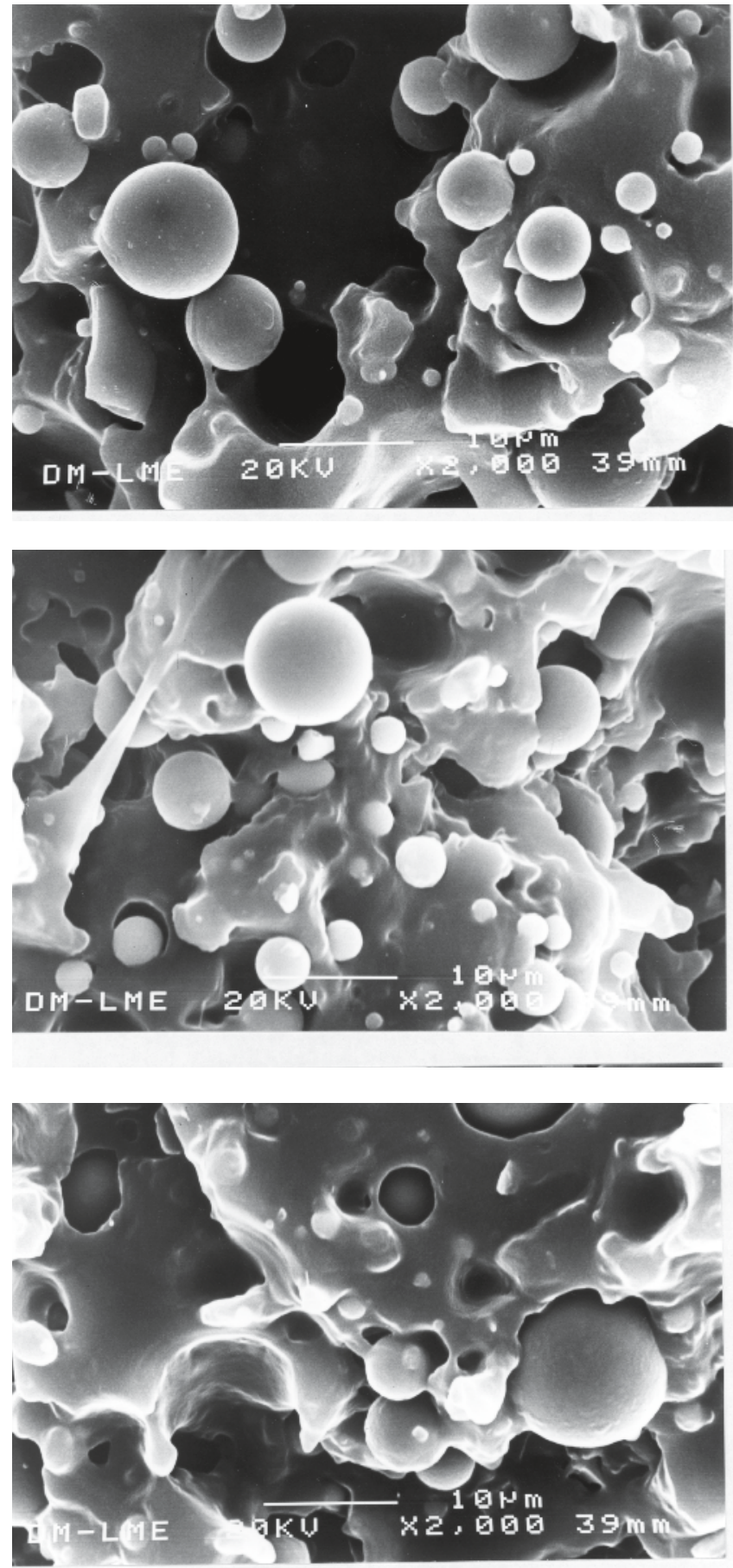

Figura 3. Micrografías por SEM de las superficies de fractura de a) B50, b) BUC50, c)BZ50; donde puede apreciarse la mayor adhesión entre EPDM y microesferas de vidrio

TABlA II. RESUlTAdOS DE LOS PARÁMETROS DE LA FRACTURA, Y DEL FACTOR DE FORMA

\begin{tabular}{|c|c|c|c|}
\hline Compuesto & $\begin{array}{c}\mathbf{w}_{\mathbf{e}} \\
\left(\mathbf{K J} / \mathbf{m}^{2}\right)\end{array}$ & $\beta$ & $\begin{array}{c}\mathbf{w}_{\mathbf{p}} \\
\left(\mathbf{M J} / \mathbf{m}^{3}\right)\end{array}$ \\
\hline B50 & 15.56 & 0.310 & 6.67 \\
BUC50 & 14.14 & 0.212 & 6.84 \\
BZ50 & 14.04 & 0.177 & 20.92 \\
\hline
\end{tabular}



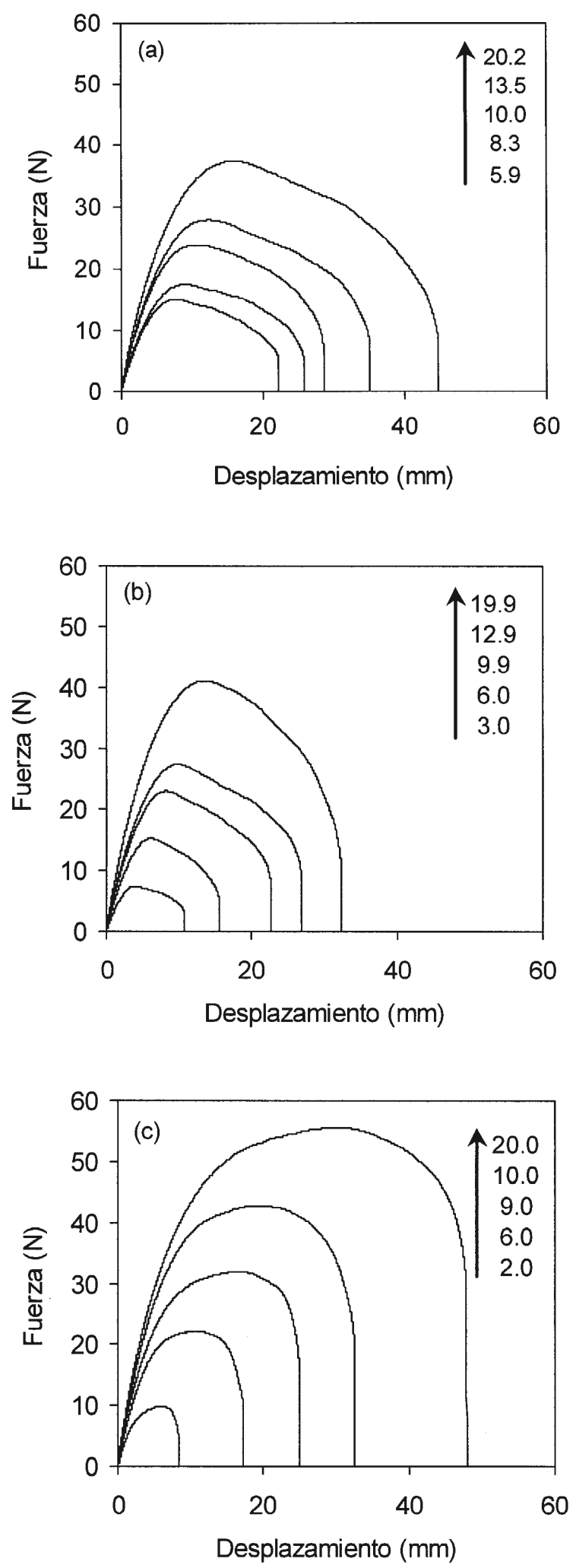

Figura 4. Curvas obtenidas en los ensayos EWF para los compuestos cargados con microesferas de vidrio; a) B50, b) BUC50, c) BZ50. Los valores junto a la flecha indican la longitud de ligamento correspondiente a cada curva. silanos con respecto a las no tratadas, pues el módulo de Young se determina en la región de pequeñas deformaciones, donde se acepta una total adhesión entre partículas y matriz en todos los compuestos.

Los valores de $\sigma_{200 \%}$ muestran que la resistencia aumenta sólo cuando la fase rígida está tratada con silanos, en particular con el Z-6032, resultando los valores $\sigma_{200 \%}$ del compuesto con microesferas no tratadas inferiores a los de la matriz elastomérica. Esto se debe a la no existencia de una interfase resistente entre la carga rígida y la matriz en el compuesto B50. En la Fig. 3a no se observa ningún tipo de adhesión entre el vidrio y la matriz en este compuesto. Los resultados hacen pensar que sí que existiría una mejor adhesión en el compuesto BUC50, pero la observación por SEM no soporta tal resultado (Fig. 3b). Sin embargo, no hay duda de la buena adhesión conseguida entre ambas fases en el compuesto BZ50 (Fig. 3c), indicándonos que el silano Z-6032 presenta un buen efecto como promotor de la adhesión entre este grado de EPDM y las microesferas de vidrio, generando en el compuesto una interfase resistente.

Al igual que sucede con el módulo de Young, a partir de temperaturas de procesado de $200{ }^{\circ} \mathrm{C}$, los valores de la resistencia descienden de manera acusada, debido probablemente a efectos de degradación del polímero.

\subsection{Comportamiento a fractura}

En la Fig. 4 se muestran ejemplos de las curvas fuerza-desplazamiento obtenidas con cada compuesto, a partir de las cuales se aplicó la teoría del trabajo esencial de fractura. Durante los ensayos se pudo observar la forma elíptica de la zona plástica desarrollada sobre el ligamento, a partir de la cual se obtuvieron los valores del factor de forma $\beta$, presentados en la Tabla II. Los valores del trabajo total específico $\left(\mathrm{w}_{\mathrm{f}}\right)$ fueron representados frente a la longitud de ligamento (Fig. 5a), de cuya linealización se obtuvieron los valores de $\mathrm{w}_{\mathrm{e}} \mathrm{y} \mathrm{w}_{\mathrm{p}}$ recogidos asimismo en la Tabla II.

En primer lugar, como era de esperar, no se observó linealidad entre el trabajo específico de fractura y la longitud de ligamento en el EPDM no cargado (Fig. 5b). Ello se debe a su carácter exclusivamente elástico. Sin embargo, por lo que se observó en los compuestos cargados, parece que la incorporación de microesferas de vidrio al EPDM introduce una contribución de deformación plástica en este elastómero, debida principalmente a fenómenos de despegue entre ambas fases.

A tenor de la forma de las curvas fuerza-desplazamiento, los compuestos parecen seguir la tendencia de que a una mayor adhesión entre la fase rígida y la fase elastomérica la fractura se hace más inestable, mostrándose ésto como una caída más brusca de la fuerza al final del ensayo, en vez de una caída suave, asociada a un puro desgarro dúctil y estable del compuesto.

El valor más elevado de trabajo específico no esencial (o plástico) de fractura encontrado en el compuesto BZ50 refuerza los resultados obtenidos de resistencia a la tracción, pues la adhesión interfacial en este compuesto es la mayor de todas, lo que contribuye a dificultar la deformación plástica del EPDM, y por tanto a necesitar más energía, incrementándose así el valor de $\mathrm{w}_{\mathrm{p}}$.

En cuanto al trabajo específico esencial de fractura, no se han encontrado diferencias significativas para las distintas muestras cargadas, lo que parece indicar que la resistencia de la 

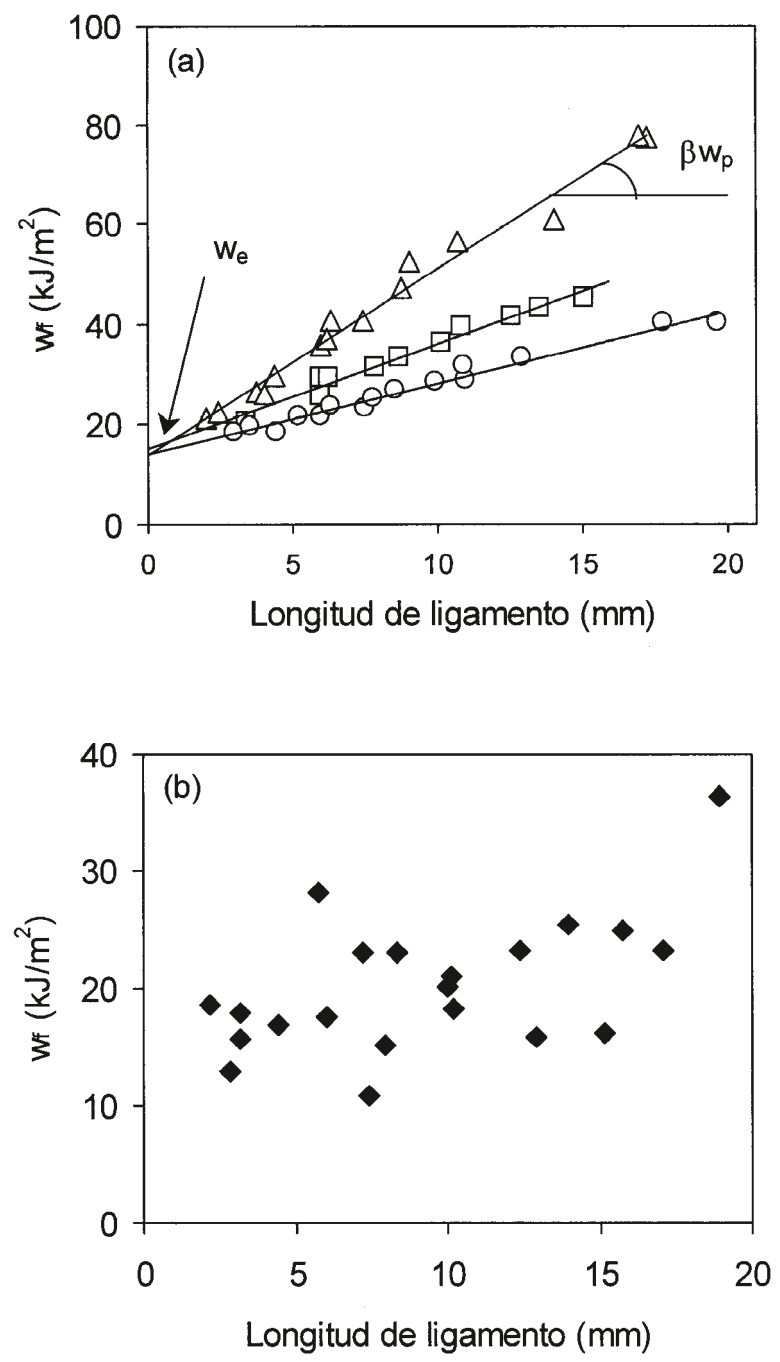

Figura 5. Linealización de los valores del trabajo específico de fractura para a) los materiales cargados y b) EPDM no cargado.

interfase no interviene significativamente en la propagación de la grieta. La propagación en todos los casos transcurre a través de la matriz de EPDM.

Como puede comprobarse en la Fig. 6, el compuesto B50 se acerca a un estado puro de tenisión plana, pero en general los 3 materiales están sometidos a estados mixtos de tensión con diferente contribución relativa de tensión plana y deformación plana, por lo que los valores de $\mathrm{w}_{\mathrm{e}}$ obtenidos deben ser tomados con cautela. Estas diferencias en el estado tensional serían debidas a la diferente adhesión entre las microesferas y el EPDM en las distintas muestras, de tal forma que al aumentar el grado de adhesión nos alejamos del estado de tensión plana, lo que contribuye a una mayor inestabilidad de la fractura al final del ensayo, tal y como se ha observado.

\section{CONCLUSIONES}

La incorporación de microesferas de vidrio al EPDM permite estudiar su comportamiento a la fractura aplicando el concepto del EWF sobre la geometría DDENT. La presencia de las partículas favorece la plasticidad del EPDM, siendo el princi-
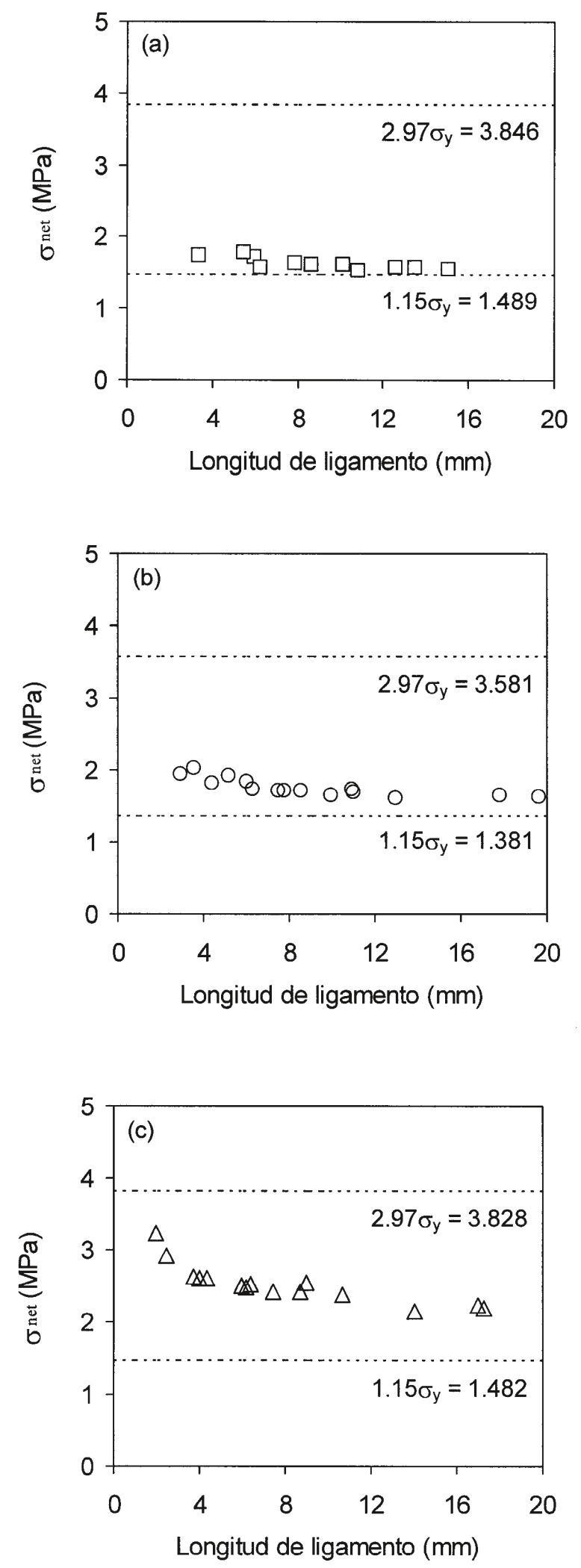

Figura 6. Comparación de los valores de la tensión máxima en los ensayos EWF con los del criterio de Hill para tensión plana y deformación plana, a) B50, b) BUC50, c) BZ50. 
pal mecanismo el despegue de la matriz. Mediante tratamiento superficial con silanos se puede incrementar el grado de adhesión entre el EPDM y el vidrio. El resultado es un aumento de la resistencia a la tracción y del trabajo plástico de fractura del compuesto, así como una mayor inestabilidad en la fractura.

Del criterio de Hill se extrae que todos los compuestos se encuentran en un estado tensional mixto, si bien más cercanos a un estado de tensión plana (sobre todo el compuesto B50) que de deformación plana. Ello hace que los resultados obtenidos mediante la aplicación del método del trabajo tengan que ser tomados con cautela.

Por encima de una temperatura de procesado de $200{ }^{\circ} \mathrm{C}$ las propiedades mecánicas de estos compuestos se resintieron de manera acusada.

\section{AGRADECIMIENTOS}

D. Arencón y C. Morhain agradecen a la Comissió Interdepartamental de Investigació i Tecnologia de Catalunya (CIRIT) la concesión de una beca predoctoral.

\section{BIBLIOGRAFÍA}

1. K.B. Broberg, "Critical review of some theories in fracture mechanics", Int. J. Fracture, 4, 11-18 (1968)

2. R. Hill, "On discontinous plastic states, with special reference to localized necking in thin sheets", J. Mech. Phys. Solids, 1, 19-30 (1952)

3. Y. W. Mai, B. Cotterell, R. Horlyck, G. Vigna, “The essential work of fracture of plane stress ductile fracture of linear polyethylenes", Pol. Engng. Sci., 27, 804809 (1987)

4. A. S. Saleemi, J. A. Nairn, "The plane-strain essential work of fracture as a measure of the fracture toughness of ductile polymers", Pol. Engng. Sci., 30, 211-218 (1990)

5. W. Y. F. Chan, J. G. Wlliams, “Determination of the fracture toughness of polymeric films by the essential work of fracture method", Polymer, 35, 1666-1672 (1994)

6. J. Karger-Kocsis, T. Czigány, "On the essential and non-essential work of fracture of biaxial-oriented filled PET film", Polymer, 37, 2433-2437 (1996)

7. B. Cotterell, J. K. Reddell, "The essential work of plane stress ductile fracture", Int. J. Fracture, 13, 267-277 (1977)

8. A.H. Priest, B. Holmes, "A multi-test piece approach to the fracture characterisation of line pipe steels", Int. J. Fracture, 17, 277-299 (1981)

9. D. E. Mouzakis, M. Gahleitner, J. Karger-Kocsis, “Toughness assessment of elastomeric PP (ELPP) by the essential work of fracture method", J. Appl. Pol. Sci., 70, 873-881 (1998)

10. D. E. Mouzakis, F. Stricker, R. Mülhaupt, “Fracture behaviour of polypropylene/glass bead/elastomer composites by using the essential work of fracture method", J. Mat. Sci., 33, 2551-2560 (1998)

11. Test protocol for essential work of fracture. European Structural Integrity Society (ESIS). Version 5, October 1997 